Theory and practise of the g-index

Peer-reviewed author version

EGGHE, Leo (2006) Theory and practise of the g-index. In: SCIENTOMETRICS, 69(1). p. 131-152.

DOI: $10.1007 / \mathrm{s} 11192-006-0144-7$

Handle: http://hdl.handle.net/1942/981 


\section{Theory and practise of the}

\section{g-index}

by

\section{Egghe ${ }^{1(*), 2}$}

1 Universiteit Hasselt (UHasselt), Campus Diepenbeek, Agoralaan, B-3590 Diepenbeek, Belgium $^{1}$

2 Universiteit Antwerpen (UA), Campus Drie Eiken, Universiteitsplein 1, B-2610 Wilrijk, Belgium

leo.egghe@uhasselt.be

\section{ABSTRACT}

The g-index is introduced as an improvement of the h-index of Hirsch to measure the global citation performance of a set of articles. If this set is ranked in decreasing order of the number of citations that they received, the g-index is the (unique) largest number such that the top $g$ articles received (together) at least $\mathrm{g}^{2}$ citations. We prove the unique existence of $\mathrm{g}$ for any set of articles and we have that $\mathrm{g} \geq \mathrm{h}$.

The general Lotkaian theory of the g-index is presented and we show that

$$
g=\left(\frac{\alpha-1}{\alpha-2}\right)^{\frac{\alpha-1}{\alpha}} T^{\frac{1}{\alpha}}
$$

\footnotetext{
${ }^{(*)}$ Permanent address.
}

Key words and phrases: g-index, h-index, Lotka, citation performance, Price medallist.

Acknowledgement: The author is grateful to Drs. M. Goovaerts for the preparation of the citation data of the Price medallists (January 2006). 
where $\alpha>2$ is the Lotkaian exponent and where $\mathrm{T}$ denotes the total number of sources.

We then present the g-index of the (still active) Price medallists for their complete careers up to 1972 and compare it with the h-index. It is shown that the g-index inherits all the good properties of the h-index and, in addition, better takes into account the citation scores of the top articles. This yields a better distinction between and order of the scientists from the point of view of visibility.

\section{Introduction}

Recently the physicist Hirsch (see Hirsch (2005)) introduced the so-called h-index - see also Ball (2005), Braun, Glänzel and Schubert (2005), Glänzel (2006a,b), Egghe and Rousseau (2006). For any general "set of papers” one can arrange these papers in decreasing order of the number of citations they received. The h-index is then the largest rank $h=r$ such that the paper on this rank (and hence also all papers on rank $1, \ldots, \mathrm{h}$ ) has h or more citations. Hence the papers on ranks $h+1, h+2, \ldots$ have not more than $h$ citations.

Although introduced by a physicist, this new science indicator has been well-received in scientometrics (informetrics). In the above mentioned references it was argued that the hindex is a simple single number incorporating publication as well as citation data (hence comprising quantitative as well as qualitative or visibility aspects) and hence has an advantage over numbers such as "number of significant papers" (which is arbitrary) or "number of citations to each of the (say) q most cited papers" (which again is not a single number). The hindex is also robust in the sense that it is insensitive to a set of uncited (or lowly cited) papers but also it is insensitive to one or several outstandingly highly cited papers. This last aspect can be considered as a drawback of the h-index. Let us discuss this point further.

Highly cited papers are, of course, important for the determination of the value $h$ of the hindex. But once a paper is selected to belong to the top h papers, this paper is not "used" any more in the determination of $\mathrm{h}$, as a variable over time. Indeed, once a paper is selected to the top group, the h-index calculated in subsequent years is not at all influenced by this paper's 
received citations further on: even if the paper doubles or triples its number of citations (or even more) the subsequent h-indexes are not influenced by this. We think it is an advantage of the h-index not to take into account the "tail” papers (with low number of citations) but it should (being a measure of overall citation performance) take into account the citation evolution of the most cited papers!

In order to overcome this disadvantage, whilst keeping the advantages of the h-index, we make the following remark: by definition of the h-index, the papers on rank $1, \ldots$, h each have at least $\mathrm{h}$ citations, hence these $\mathrm{h}$ papers together have at least $\mathrm{h}^{2}$ citations. But it could well be (see examples further on) that the first $h+1$ papers have together $(h+1)^{2}$ or more citations (here we use the fact that, most probably, the top papers have much more than h citations) and the same might be true for ranks $h+2$ (the top $(h+2)$ papers having together at least $(\mathrm{h}+2)^{2}$ citations) or even higher.

Therefore, in the Letter Egghe (2006a) we introduced a simple variant of the h-index: the gindex.

\section{Definition I.1:}

A set of papers has a g-index $\mathrm{g}$ if $\mathrm{g}$ is the highest rank such that the top g papers have, together, at least $\mathrm{g}^{2}$ citations. This also means that the top $\mathrm{g}+1$ papers have less than $(\mathrm{g}+1)^{2}$ papers.

The following proposition (also remarked in Egghe (2006a)) is trivial.

\section{Proposition I.2:}

In all cases one has that

$$
\mathrm{g} \geq \mathrm{h}
$$

\section{Proof:}

Since $h$ satisfies the requirement that the top $h$ papers have at least $h^{2}$ papers and since $g$ is the largest number with this property, it is clear that $\mathrm{g} \geq \mathrm{h}$. 
An example shows the easy calculation of the h-index and the g-index. The data are the author's own citation data derived from the Web of Knowledge (WoK). It must be underlined, however, that the real citation data can be much higher due to several reasons:

- $\quad$ only source journals, selected by Thomson ISI are used,

- $\quad$ unclear citations (even to source journals, e.g. "to appear” etc.) are not counted in the WoK.

In the table below TC stands for the total number of citations for each paper on rank $r=1,2, \ldots$ and $\Sigma$ TC stands for the cumulative number of citations to the papers on rank 1,..,r (for each $\mathrm{r}$ ). The bold face typed numbers give the explanation for the $\mathrm{h}$-index $\mathrm{h}=13$ and the g-index $\mathrm{g}=19$. Indeed $\mathrm{h}=13$ is the highest rank such that all papers on rank $1, \ldots, \mathrm{h}$ have at least 13 citations (and hence the papers on rank 14 or higher have not more than 13 citations). Also $\mathrm{g}=19$ is the highest rank such that the top 19 papers have at least $19^{2}=361$ citations (here $381>361$ ); on rank 20 we have $392<20^{2}=400$ citations.

Table 1. Ranking of the papers of L. Egghe according to their number of citations received (source: WoK).

\begin{tabular}{cccc} 
TC & $r$ & $\Sigma T C$ & $r^{2}$ \\
& \multicolumn{1}{r}{4} & & \\
47 & 1 & 47 & 1 \\
42 & 2 & 89 & 4 \\
37 & 3 & 126 & 9 \\
36 & 4 & 162 & 16 \\
21 & 5 & 183 & 25 \\
18 & 6 & 201 & 36 \\
17 & 7 & 218 & 49 \\
16 & 8 & 234 & 64 \\
16 & 9 & 250 & 81 \\
16 & 10 & 266 & 100 \\
15 & 11 & 281 & 121 \\
13 & 12 & 294 & 144 \\
\cline { 1 - 1 } 13 & 13 & 307 & 169 \\
\cline { 1 - 1 } 13 & 14 & 320 & 196 \\
13 & 15 & 333 & 225 \\
12 & 16 & 345 & 256 \\
12 & 17 & 357 & 289 \\
12 & 18 & 369 & 324 \\
\cline { 2 - 3 } 12 & $\mathbf{1 9}$ & $\mathbf{3 8 1}$ & $\mathbf{3 6 1}$ \\
\hline 11 & 20 & 392 & 400 \\
& & &
\end{tabular}


In the last section of this article we will compare the h- and g-indexes of the (active) Price medallists (updated calculations of the h-index as in Glänzel and Persson (2005) and new calculations of the g-index) showing the advantage of the g-index above the h-index but in the next section we will give the mathematical theory of the g-index based on Lotka's law

$$
f(j)=\frac{C}{j^{\alpha}}
$$

$\mathrm{j} \geq 1, \mathrm{C}>0, \alpha>2$ (it will turn out that, if we let $\mathrm{j}$ to be arbitrary large - which we assume here for the sake of simplicity - we need to take $\alpha>2)$. In case of (2) we will show that ( $\mathrm{T}=$ total number of sources (= papers here))

$$
g=\left(\frac{\alpha-1}{\alpha-2}\right)^{\frac{\alpha-1}{\alpha}} \mathrm{T}^{\frac{1}{\alpha}}
$$

, hence by Glänzel (2006b) or Egghe and Rousseau (2006), since one showed there that $\mathrm{h}=\mathrm{T}^{\frac{1}{\alpha}}$, we have

$$
g=\left(\frac{\alpha-1}{\alpha-2}\right)^{\frac{\alpha-1}{\alpha}} h>h
$$

Also the relation of $\mathrm{g}$ with the total number A of items (= citations here) is given. Before this theory is developed we will, firstly, show the general existence theorem for the g-index: for any set of papers we always have that the g-index exists and is unique.

Note, cf. Braun, Glänzel and Schubert (2005), Egghe (2006a), Egghe and Rousseau (2006), that any set of papers can be taken here, e.g. the papers of a scientist but also a year's production (articles) in a journal can be used. 


\section{Mathematical theory of the g-index}

First we will give a mathematically exact definition of the g-index in continuous variables.

\section{II.1 Mathematical definition of the g-index}

Let $f(j)(j \geq 1)$ denote the general size-frequency function of the system (which can be more general than the papers-citation relation: we can work in general information production processes (IPPs) where we have sources that produce items - cf. Egghe and Rousseau (1990), Egghe (2005)). We do not suppose $\mathrm{f}$ to be Lotkaian at this moment. Let $g(r)(r \in[0, T])$ denote the general rank-frequency function (the function $g(r)$ should not be confused with the gindex; we keep the $f(j)$ and $g(r)$ notation since this has been done in all previous articles and books on this topic - throughout the text it will be clear whether we deal with the function $g(r)$ or with the g-index g). The general (defining) relation between the functions $f(j)$ and $\mathrm{g}(\mathrm{r})$ is as follows:

$$
r=g^{-1}(j)=\int_{j}^{\infty} f\left(j^{\prime}\right) d j^{\prime}
$$

Indeed, if $r=g^{-1}(j)$ (the inverse of the function $g(r)$ ) then $g(r)=j$ and there are $r$ sources with an item density value larger than or equal to j. Denote

$$
\mathrm{G}(\mathrm{r})=\int_{0}^{\mathrm{r}} \mathrm{g}\left(\mathrm{r}^{\prime}\right) \mathrm{dr} \mathrm{r}^{\prime}
$$

the cumulative number of items in the sources up to rank r (i.e. the top r sources).

\section{Definition:}

The rank $r$ is the g-index: $r=g$ of this system if $r$ is the highest value such that

$$
\mathrm{G}(\mathrm{r}) \geq \mathrm{r}^{2}
$$


Note that this is the exact formulation of the g-index as proposed in Section I in practical systems.

\section{II.2 Existence theorem for the g-index}

\section{Theorem II.2.1}

Every general system has a unique g-index.

\section{Proof:}

Define, for all $r \in] 0, T]$

$$
H(r)=\frac{G(r)}{r}
$$

and we define $\mathrm{H}(0)=\lim _{\mathrm{r} \rightarrow 0} \mathrm{H}(\mathrm{r})=\mathrm{g}(0)$. We first prove that $\mathrm{H}$ strictly decreases on $[0, \mathrm{~T}]$. Indeed

$$
H^{\prime}(r)=\frac{\operatorname{rg}(r)-G(r)}{r^{2}}<0
$$

since

$$
\operatorname{rg}(\mathrm{r})<\mathrm{G}(\mathrm{r})=\int_{0}^{\mathrm{r}} \mathrm{g}\left(\mathrm{r}^{\prime}\right) \mathrm{dr}
$$

since the function $g$ is strictly decreasing (by (5)) for all values of $r \in] 0, T]$. Since $H(0)=\lim _{r \rightarrow 0} H(r)$ we hence have that $H$ strictly decreases on $[0, T]$. If $H(T) \geq T$ then $G(T) \geq T^{2}$ and since this is the largest possible value, we have the unique g-index $g=T$. Suppose now that $\mathrm{H}(\mathrm{T})<\mathrm{T}$. Define

$$
\begin{aligned}
& F(r)=H(r)-r \\
& F(r)=\frac{G(r)}{r}-r
\end{aligned}
$$


Since $\mathrm{H}(0)>0$ (since the function g strictly decreases (by (5)) and by (8)) and $\mathrm{H}(\mathrm{T})<\mathrm{T}$ we have $F(0)>0$ and $F(T)<0$. Hence, since $F$ is continuous, there is a value $r$ such that $\mathrm{F}(\mathrm{r})=0$. By (8) and (9) we hence have the existence of a value $\mathrm{r}$ such that

$$
\mathrm{G}(\mathrm{r})=\mathrm{r}^{2}
$$

Note that this satisfies (7) and that it is the highest possible value that satisfies (7): indeed, $\mathrm{H}$ strictly decreases, so, for every value $r$ ' $>r$ we have

$$
\mathrm{H}\left(\mathrm{r}^{\prime}\right)<\mathrm{H}(\mathrm{r})
$$

By (8):

$$
\begin{aligned}
& \frac{G\left(r^{\prime}\right)}{r^{\prime}}<\frac{G(r)}{r}=r \\
& G\left(r^{\prime}\right)<r^{\prime}<r^{\prime 2}
\end{aligned}
$$

contradicting (7). Hence this unique $r$ value is the g-index: $r=g$. Note that, except if $G(T) \geq T^{2}$, we can prove that the g-index always satisfies (7) with an equality sign instead of $\geq$.

Now we will give formulae for the g-index in terms of parameters that appear in Lotkaian informetrics.

\section{II.3 Formulae for the g-index in Lotkaian systems}

If $\mathrm{G}(\mathrm{T}) \leq \mathrm{T}^{2}$ then we know from the proof of Theorem II.2.1 that the g-index satisfies (7) with an equality sign:

$$
\mathrm{G}(\mathrm{g})=\mathrm{g}^{2}
$$


Otherwise (if $G(T)>T^{2}$ ) we take $g=T$.

We have the following theorem.

\section{Theorem II.3.1:}

Given the law of Lotka

$$
f(j)=\frac{C}{j^{\alpha}}
$$

$\mathrm{j} \geq 1, \mathrm{C}>0, \alpha>2$, we have that the g-index equals

$$
g=\left(\frac{\alpha-1}{\alpha-2}\right)^{\frac{\alpha-1}{\alpha}} T^{\frac{1}{\alpha}}
$$

if $\left(\frac{\alpha-1}{\alpha-2}\right)^{\frac{\alpha-1}{\alpha}} \mathrm{T}^{\frac{1}{\alpha}} \leq \mathrm{T}$ and $\mathrm{g}=\mathrm{T}$ if $\left(\frac{\alpha-1}{\alpha-2}\right)^{\frac{\alpha-1}{\alpha}} \mathrm{T}^{\frac{1}{\alpha}}>\mathrm{T}$.

Here $\mathrm{T}$ denotes the total number of sources.

\section{Proof:}

First proof:

The first (cf. (5))

$$
\begin{aligned}
r=g^{-1}(j) & =\int_{j}^{\infty} f\left(j^{\prime}\right) d j^{\prime} \\
& =\frac{C}{\alpha-1} j^{1-\alpha}
\end{aligned}
$$

sources yield a total number of items (since $\alpha>2$ )

$$
\int_{j}^{\infty} j^{\prime} f\left(j^{\prime}\right) d j^{\prime}=\frac{C}{\alpha-2} j^{2-\alpha}
$$


(cf. also Egghe (2005), Chapter II).

So, by (10) we have $r=g$ if

$$
\frac{C}{\alpha-2} j^{2-\alpha}=g^{2}
$$

and if this g satisfies $\mathrm{g} \leq \mathrm{T}$ (otherwise take $\mathrm{g}=\mathrm{T}$ ).

By (13):

$$
j=\left(\frac{(\alpha-1) r}{C}\right)^{\frac{1}{1-\alpha}}
$$

(16) (for $r=g$ ) in (15) yields

$$
\begin{aligned}
& g=\left[\frac{C}{\alpha-2}\left(\frac{\alpha-1}{C}\right)^{\frac{\alpha-2}{\alpha-1}}\right]^{\frac{\alpha-1}{\alpha}} \\
& g=\left(\frac{\alpha-1}{\alpha-2}\right)^{\frac{\alpha-1}{\alpha}} T^{\frac{1}{\alpha}}
\end{aligned}
$$

using that $T=\frac{C}{\alpha-1}$ as follows from (13) by taking $j=1$. This value is taken as the $g$ index if it is $\leq \mathrm{T}$ and we take $\mathrm{g}=\mathrm{T}$ if it is strictly larger than $\mathrm{T}$.

Second proof:

Now we work directly with formula (10). Note that Lotka's law (11) is equivalent with Zipf's law

$$
g(r)=\frac{B}{r^{\beta}}
$$


$B, \beta>0, r \in] 0, T]$ where we have the relations

$$
\begin{gathered}
B=\left(\frac{C}{\alpha-1}\right)^{\frac{1}{\alpha-1}} \\
\beta=\frac{1}{\alpha-1}
\end{gathered}
$$

(cf. Egghe (2005), Exercise II.2.2.6 but see also the Appendix in Egghe and Rousseau (2006) where a proof is given.).

Note that by (19) $\alpha>2$ is equivalent with $0<\beta<1$. If that is the case, (10) gives

$$
\int_{0}^{g} \frac{B}{r^{\beta}} d r=g^{2}
$$

hence, since $0<\beta<1$

$$
\frac{B}{1-\beta} g^{1-\beta}=g^{2}
$$

Hence

$$
g=\left(\frac{B}{1-\beta}\right)^{\frac{1}{\beta+1}}
$$

Now (18) and (19) in (20) again yield formula (12).

\section{Corollary II.3.2:}

If $\mathrm{g}$ is the g-index and $\mathrm{h}$ is the h-index of a Lotkaian system with exponent $\alpha>2$, then

$$
g=\left(\frac{\alpha-1}{\alpha-2}\right)^{\frac{\alpha-1}{\alpha}} h
$$


(if this value is $\leq \mathrm{T}$; otherwise $\mathrm{g}=\mathrm{T}$ ).

\section{Proof:}

This follows readily from (12) and the fact that $\mathrm{h}=\mathrm{T}^{\frac{1}{\alpha}}$, see Egghe and Rousseau (2006) (also proved, approximatively, in Glänzel (2006b)).

Taking $\mathrm{j}=1$ in (13) and (14) we see that the total number of sources $\mathrm{T}$ equals $\frac{\mathrm{C}}{\alpha-1}$ and that the total number of items A equals $\frac{C}{\alpha-2}$, hence

$$
\mu=\frac{\mathrm{A}}{\mathrm{T}}=\frac{\alpha-1}{\alpha-2}
$$

equals the average number of items per source (cf. also Egghe (2005), Chapter II). Hence we have the following corollary

\section{Corollary II.3.3:}

If $\mu$ is as above we have in case of (21)

$$
g=\mu^{\frac{\alpha-1}{\alpha}} h
$$

The g-index in function of $\alpha$ and $\mathrm{A}$ is as in Corollary II.3.4.

\section{Corollary II.3.4:}

We have

$$
g=\left(\frac{\alpha-1}{\alpha-2}\right)^{\frac{\alpha-2}{\alpha}} \mathrm{A}^{\frac{1}{\alpha}}
$$

(if this value is $\leq \mathrm{T}$ ). 


\section{Proof:}

This follows readily from (22) and (12).

We can also determine the item density $\mathrm{j}$ for which we have $\mathrm{r}=\mathrm{g}$. In practical cases this means the number of items in the source at rank g. Note that this is $h$ for the h-index $r=h$, by definition of the Hirsch index.

For the g-index we have: if the value in (12) is $>\mathrm{T}$ we have $\mathrm{j}=\mathrm{g}(\mathrm{T})=1$ and if the value in (12) is $\leq \mathrm{T}$ we substitute $\mathrm{r}=\mathrm{g}$ in (17), using (18) and (19) and the fact that $\mathrm{T}=\frac{\mathrm{C}}{\alpha-1}$, yielding

$$
\mathrm{j}=\left(\frac{\alpha-2}{\alpha-1} \mathrm{~T}\right)^{\frac{1}{\alpha}}
$$

We immediately see that $\mathrm{j}<\mathrm{h}$ which is logical since $\mathrm{g}>\mathrm{h}$ and the item density is $\mathrm{h}$ in case $\mathrm{r}=\mathrm{h}$. Formula (26) presents a concrete formula for the item density cut-off place.

Note that, although $\alpha$ can be any value $\alpha>2$, we do not have $\lim _{\substack{\alpha \rightarrow 2 \\>}} j=0$. Indeed since the validity of (12) is limited to $\mathrm{g} \leq \mathrm{T}$ we have, by (12) that

$$
\left(\frac{\alpha-1}{\alpha-2}\right)^{\frac{\alpha-1}{\alpha}} \mathrm{T}^{\frac{1}{\alpha}} \leq \mathrm{T}
$$

from which it follows that

$$
\mu=\frac{\mathrm{A}}{\mathrm{T}}=\frac{\alpha-1}{\alpha-2} \leq \mathrm{T}
$$

This implies in (26) that 


$$
j=\left(\frac{T^{2}}{A}\right)^{\frac{1}{\alpha}} \geq 1
$$

, by (27).

The case

$$
\left(\frac{\alpha-1}{\alpha-2}\right)^{\frac{\alpha-1}{\alpha}} \mathrm{T}^{\frac{1}{\alpha}}>\mathrm{T}
$$

(hence where we take $\mathrm{g}=\mathrm{T}$ ) occurs in the following case: from (28) it follows that

$$
\frac{\alpha-1}{\alpha-2}>\mathrm{T}
$$

By (22) we have

$$
\mu=\frac{\mathrm{A}}{\mathrm{T}}>\mathrm{T}
$$

hence

$$
\mathrm{A}>\mathrm{T}^{2}
$$

Equivalently, (29) gives the condition in $\alpha$ :

$$
\alpha<\frac{2 \mathrm{~T}-1}{\mathrm{~T}-1}
$$

Note that

$$
\frac{2 \mathrm{~T}-1}{\mathrm{~T}-1}>2
$$


so that (31) can occur (with the condition $\alpha>2$ ).

\section{Remark II.3.5:}

It might seem strange that $\mathrm{g}=\mathrm{T}$ is possible in this Lotkaian model. Note however that (22) implies that

$$
\alpha=\frac{2 \mathrm{~A}-\mathrm{T}}{\mathrm{A}-\mathrm{T}}
$$

So, for every T fixed, if we let $\mathrm{A} \rightarrow \infty$ we have that $\alpha \rightarrow 2$ (but $\alpha>2$ ) so that we are within the limitations of our theory. In this case we have

$$
A=G(T)>T^{2}
$$

and hence $\mathrm{g}=\mathrm{T}$.

In the next section we will apply the g-index to the publications and citations of the (still active) Price medallists and compare these g-indexes with the h-indexes of these same data.

\section{Calculation and comparison of the $h$ - and g- indexes of the (still active) Price medallists}

In Glänzel and Persson (2005), the h-indexes for the (still active) Price medallists are calculated. We could use these numbers and compare them with the here defined g-index. However for this we need to extend the tables in Glänzel and Persson (2005) (since g $\geq \mathrm{h}$ ) and it is hardly impossible to do this since we should do this for the maximal citing time August 2005 (since then the tables in Glänzel and Persson (2005) were produced). So the easiest thing to do is to remake these tables for the present time (January 2006) and make them long enough so that, on the same tables, the h- as well as the g-index can be calculated. 
We have opted not to limit the publication year to 1986 or higher (as was the case in Glänzel and Persson (2005)). Indeed, the h-indexes in Glänzel and Persson (2005) seemed a bit unnatural in several senses. E. Garfield did not have the highest h-index (which we, normally, could expect) and H. Small scored lowest of all the Price medallists. The major reason for these observations is that, by limiting the publication year to 1986 or higher, one cuts away most publications (and perhaps the highest cited ones) of the relatively older scientists. Since we want to make a comparison of scientists (and not to draw conclusions on informetrics fields) we decided not to limit the publication year (except to the evident limit 1972 since before that date the ISI (now Thomson ISI) data do not exist.

For the same reason we count all publications even if scientists have published in different domains (e.g. T. Braun in chemistry and L. Egghe in mathematics). These publications were not used in the Glänzel and Persson study.

Of course, by not limiting the publication period and the publication field, one might argue that there is a bias towards the older scientists. This is true but, with the h- and g-indexes, we want to indicate the "overall performance (visibility)” of the scientists as they are viewn today (in the sense of "lifetime achievement").

We base ourselves on the Web of Knowledge (WoK) and hence we are limited to the Thomson ISI data. This means that no citations to non-source journals or conference proceedings articles or books are counted. In addition, no citations to incomplete references are counted even if they are to source journal articles (e.g. a citation to JASIST, 2001, to appear): these are not collected in the WoK “times cited” data. So the actual h- and g-indexes can be somewhat higher but this effect plays for every scientist so that comparisons are still possible and also these limitations do not jeopardise the possibility to compare the h- and gindex.

The tables of citation data of the (still active) medallists are found in the Appendix. The table stops one line below the g-index since this is all we need. The number $r$ denotes the rank of the publication and TC denotes the total number of citations to the paper on rank r. The number $\Sigma$ TC denotes the cumulative number of citations to the first $\mathrm{r}$ ranked papers. Finally, also the table of $r^{2}$ values is presented as well as the publication year (PY) of the article on 
rank $\mathrm{r}$. The h- and g-index determination is highlighted in the tables in the Appendix. Table 2 gives the results in decreasing order of $\mathrm{h}$ and $\mathrm{g}$.

Table 2. h- and g-indexes of Price medallists in decreasing order

\begin{tabular}{|c|c|}
\hline Name & h-index \\
\hline Garfield & 27 \\
Narin & 27 \\
Braun & 25 \\
Van Raan & 19 \\
Glänzel & 18 \\
Moed & 18 \\
Schubert & 18 \\
Small & 18 \\
Martin & 16 \\
Egghe & 13 \\
Ingwersen & 13 \\
Leydesdorff & 13 \\
Rousseau & 13 \\
White & 12 \\
& \\
\hline
\end{tabular}

\begin{tabular}{|c|c|}
\hline Name & g-index \\
\hline Garfield & 59 \\
Narin & 40 \\
Small & 39 \\
Braun & 38 \\
Schubert & 30 \\
Glänzel & 27 \\
Martin & 27 \\
Moed & 27 \\
Van Raan & 27 \\
Ingwersen & 26 \\
White & 25 \\
Egghe & 19 \\
Leydesdorff & 19 \\
Rousseau & 15 \\
\hline
\end{tabular}

We leave the detailed (subjective) interpretation of Table 2 to the reader but it is clear that the g-index column is more in line with intuition and with the raw data in the Appendix than the h-index column. In other words, the g-index, as simple as the h-index (a single measure, containing publication and citation elements), contains more comparative information from the raw data than the h-index and resembles more the overall feeling of "visibility" or "life time achievement”.

A possible interesting measure is $\frac{\mathrm{g}}{\mathrm{h}}$, i.e. the relative increase of g with respect to $\mathrm{h}$. The result is presented in Table 3, in decreasing order of $\frac{\mathrm{g}}{\mathrm{h}}$. Here we see remarkable order changes with respect to the h- or g-orderings. 
Table 3. $\frac{\mathrm{g}}{\mathrm{h}}$-values of Price medallists in decreasing order

\begin{tabular}{|l|c|}
\hline \multicolumn{1}{|c|}{ Name } & $\frac{\mathrm{g}}{\mathrm{h}}$ \\
\hline Garfield & 2.19 \\
Small & 2.17 \\
White & 2.08 \\
Ingwersen & 2.00 \\
Martin & 1.69 \\
Schubert & 1.67 \\
Braun & 1.52 \\
Glänzel & 1.50 \\
Moed & 1.50 \\
Narin & 1.48 \\
Egghe & 1.46 \\
Leydesdorff & 1.46 \\
Van Raan & 1.42 \\
Rousseau & 1.15 \\
\hline
\end{tabular}




\section{Appendix}

Tables of TC, r, sTC, $\mathrm{r}^{2}$ and PY for each of the (still active) Price medallists and determination of the $\mathrm{h}$ - and g-index.

\section{Garfield E.}

\begin{tabular}{|c|c|c|c|c|}
\hline TC & $r$ & $\Sigma \mathrm{TC}$ & $r^{2}$ & PY \\
\hline 625 & 1 & 625 & 1 & 1972 \\
\hline 149 & 2 & 774 & 4 & 1980 \\
\hline 138 & 3 & 912 & 9 & 1977 \\
\hline 132 & 4 & 1044 & 16 & 1983 \\
\hline 132 & 5 & 1176 & 25 & 1981 \\
\hline 129 & 6 & 1305 & 36 & 1979 \\
\hline 127 & 7 & 1432 & 49 & 1996 \\
\hline 111 & 8 & 1543 & 64 & 1978 \\
\hline 109 & 9 & 1652 & 81 & 1975 \\
\hline 108 & 10 & 1760 & 100 & 1985 \\
\hline 107 & 11 & 1867 & 121 & 1984 \\
\hline 105 & 12 & 1972 & 144 & 1982 \\
\hline 104 & 13 & 2076 & 169 & 1986 \\
\hline 101 & 14 & 2177 & 196 & 1976 \\
\hline 96 & 15 & 2273 & 225 & 1973 \\
\hline 91 & 16 & 2364 & 256 & 1976 \\
\hline 89 & 17 & 2453 & 289 & 1974 \\
\hline 88 & 18 & 2541 & 324 & 1986 \\
\hline 87 & 19 & 2628 & 361 & 1987 \\
\hline 85 & 20 & 2713 & 400 & 1979 \\
\hline 80 & 21 & 2793 & 441 & 1985 \\
\hline 67 & 22 & 2860 & 484 & 1988 \\
\hline 63 & 23 & 2923 & 529 & 1999 \\
\hline 41 & 24 & 2964 & 576 & 1980 \\
\hline 29 & 25 & 2993 & 625 & 1990 \\
\hline 28 & 26 & 3021 & 676 & 1987 \\
\hline 27 & 27 & 3048 & 729 & 1987 \\
\hline 26 & 28 & 3074 & 784 & 1976 \\
\hline 26 & 29 & 3100 & 841 & 1992 \\
\hline 23 & 30 & 3123 & 900 & 1978 \\
\hline 23 & 31 & 3146 & 961 & 1990 \\
\hline 20 & 32 & 3166 & 1024 & 1990 \\
\hline 19 & 33 & 3185 & 1089 & 1998 \\
\hline 19 & 34 & 3204 & 1156 & 1998 \\
\hline 18 & 35 & 3222 & 1225 & 1985 \\
\hline 18 & 36 & 3240 & 1296 & 1979 \\
\hline 18 & 37 & 3258 & 1369 & 1996 \\
\hline
\end{tabular}




$\begin{array}{rrrrr}16 & 38 & 3274 & 1444 & 1979 \\ 15 & 39 & 3289 & 1521 & 1990 \\ 14 & 40 & 3303 & 1600 & 1976 \\ 13 & 41 & 3316 & 1681 & 1973 \\ 13 & 42 & 3329 & 1764 & 1973 \\ 13 & 43 & 3342 & 1849 & 1973 \\ 13 & 44 & 3355 & 1936 & 1998 \\ 13 & 45 & 3368 & 2025 & 1990 \\ 12 & 46 & 3380 & 2116 & 1973 \\ 12 & 47 & 3392 & 2209 & 2000 \\ 12 & 48 & 3404 & 2304 & 1998 \\ 12 & 49 & 3416 & 2401 & 1997 \\ 12 & 50 & 3428 & 2500 & 1996 \\ 11 & 51 & 3439 & 2601 & 1998 \\ 11 & 52 & 3450 & 2704 & 1997 \\ 10 & 53 & 3460 & 2809 & 1985 \\ 10 & 54 & 3470 & 2916 & 1984 \\ 9 & 55 & 3479 & 3025 & 1984 \\ 9 & 56 & 3488 & 3136 & 1975 \\ 9 & 57 & 3497 & 3249 & 1972 \\ 9 & 58 & 3506 & 3364 & 2002 \\ 9 & 59 & 3515 & 3481 & 1998 \\ 9 & 60 & 3524 & 3600 & 1990\end{array}$

\section{Braun T.}

$\begin{array}{ccccc}\text { TC } & r & \Sigma T C & r^{2} & \text { PY } \\ 125 & 1 & 125 & 1 & 1978 \\ 124 & 2 & 249 & 4 & 1989 \\ 78 & 3 & 327 & 9 & 1986 \\ 66 & 4 & 393 & 16 & 1975 \\ 57 & 5 & 450 & 25 & 1974 \\ 57 & 6 & 507 & 36 & 1990 \\ 55 & 7 & 562 & 49 & 1974 \\ 51 & 8 & 613 & 64 & 1989 \\ 43 & 9 & 656 & 81 & 1992 \\ 42 & 10 & 698 & 100 & 1974 \\ 38 & 11 & 736 & 121 & 1983 \\ 37 & 12 & 773 & 144 & 1995 \\ 37 & 13 & 810 & 169 & 1994 \\ 35 & 14 & 845 & 196 & 1980 \\ 35 & 15 & 880 & 225 & 1999 \\ 33 & 16 & 913 & 256 & 1988 \\ 32 & 17 & 945 & 289 & 1995 \\ 31 & 18 & 976 & 324 & 1975 \\ 31 & 19 & 1007 & 361 & 1995 \\ 28 & 20 & 1035 & 400 & 1977 \\ 27 & 21 & 1062 & 441 & 1973\end{array}$




\begin{tabular}{llllll}
\hline 27 & 22 & 1089 & 484 & 1988 \\
27 & 23 & 1116 & 529 & 1987 \\
26 & 24 & 1142 & 576 & 2000 \\
\cline { 1 - 2 } 26 & 25 & 1168 & 625 & 1994 \\
\cline { 1 - 2 } 25 & 26 & 1193 & 676 & 1973 \\
25 & 27 & 1218 & 729 & 1972 \\
23 & 28 & 1241 & 784 & 1978 \\
23 & 29 & 1264 & 841 & 1973 \\
23 & 30 & 1287 & 900 & 1994 \\
23 & 31 & 1310 & 961 & 1987 \\
22 & 32 & 1332 & 1024 & 1983 \\
22 & 33 & 1354 & 1089 & 1982 \\
22 & 34 & 1376 & 1156 & 1980 \\
22 & 35 & 1398 & 1225 & 1987 \\
21 & 36 & 1419 & 1296 & 1973 \\
21 & 37 & 1440 & 1369 & 1973 \\
20 & 38 & 1460 & 1444 & 1982 \\
20 & 39 & 1480 & 1521 & 1982
\end{tabular}

Small H.

\begin{tabular}{|c|c|c|c|c|}
\hline TC & $r$ & $\Sigma \mathrm{TC}$ & $r^{2}$ & PY \\
\hline 305 & 1 & 305 & 1 & 1973 \\
\hline 239 & 2 & 544 & 4 & 1974 \\
\hline 127 & 3 & 671 & 9 & 1978 \\
\hline 109 & 4 & 780 & 16 & 1974 \\
\hline 86 & 5 & 866 & 25 & 1977 \\
\hline 80 & 6 & 946 & 36 & 1985 \\
\hline 77 & 7 & 1023 & 49 & 1985 \\
\hline 75 & 8 & 1098 & 64 & 1985 \\
\hline 67 & 9 & 1165 & 81 & 1999 \\
\hline 49 & 10 & 1214 & 100 & 1979 \\
\hline 44 & 11 & 1258 & 121 & 1980 \\
\hline 36 & 12 & 1294 & 144 & 1980 \\
\hline 26 & 13 & 1320 & 169 & 1981 \\
\hline 26 & 14 & 1346 & 196 & 1986 \\
\hline 25 & 15 & 1371 & 225 & 1976 \\
\hline 22 & 16 & 1393 & 256 & 1997 \\
\hline 22 & 17 & 1415 & 289 & 1993 \\
\hline 18 & 18 & 1433 & 324 & 1974 \\
\hline 18 & 19 & 1451 & 361 & 1994 \\
\hline 15 & 20 & 1466 & 400 & 1999 \\
\hline 12 & 21 & 1478 & 441 & 1986 \\
\hline 10 & 22 & 1488 & 484 & 1989 \\
\hline 9 & 23 & 1497 & 529 & 1975 \\
\hline 8 & 24 & 1505 & 576 & 1998 \\
\hline 8 & 25 & 1513 & 625 & 1987 \\
\hline
\end{tabular}




$\begin{array}{lllll}7 & 26 & 1520 & 676 & 1989 \\ 6 & 27 & 1526 & 729 & 1998 \\ 5 & 28 & 1531 & 784 & 1977 \\ 5 & 29 & 1536 & 841 & 1974 \\ 5 & 30 & 1541 & 900 & 1999 \\ 3 & 31 & 1544 & 961 & 1979 \\ 3 & 32 & 1547 & 1024 & 1995 \\ 2 & 33 & 1549 & 1089 & 1975 \\ 2 & 34 & 1551 & 1156 & 2004 \\ 2 & 35 & 1553 & 1225 & 2003 \\ 1 & 36 & 1554 & 1296 & 1973 \\ 1 & 37 & 1555 & 1369 & 2004 \\ 1 & 38 & 1556 & 1444 & 1997 \\ 1 & 39 & 1557 & 1521 & 1996 \\ 1 & 40 & 1558 & 1600 & 1992\end{array}$

\section{Van Raan A.F.J.}

\begin{tabular}{|c|c|c|c|c|}
\hline TC & $r$ & $\Sigma \mathrm{TC}$ & $r^{2}$ & PY \\
\hline 108 & 1 & 108 & 1 & 1985 \\
\hline 51 & 2 & 159 & 4 & 1996 \\
\hline 49 & 3 & 208 & 9 & 1991 \\
\hline 41 & 4 & 249 & 16 & 1985 \\
\hline 35 & 5 & 284 & 25 & 1991 \\
\hline 32 & 6 & 316 & 36 & 1973 \\
\hline 31 & 7 & 347 & 49 & 1990 \\
\hline 30 & 8 & 377 & 64 & 1990 \\
\hline 25 & 9 & 402 & 81 & 1993 \\
\hline 25 & 10 & 427 & 100 & 1974 \\
\hline 23 & 11 & 450 & 121 & 1995 \\
\hline 22 & 12 & 472 & 144 & 1998 \\
\hline 22 & 13 & 494 & 169 & 1997 \\
\hline 21 & 14 & 515 & 196 & 2000 \\
\hline 20 & 15 & 535 & 225 & 2001 \\
\hline 19 & 16 & 554 & 256 & 1998 \\
\hline 19 & 17 & 573 & 289 & 1998 \\
\hline 19 & 18 & 592 & 324 & 1994 \\
\hline 19 & 19 & 611 & 361 & 1994 \\
\hline 18 & 20 & 629 & 400 & 1998 \\
\hline 18 & 21 & 647 & 441 & 1993 \\
\hline 17 & 22 & 664 & 484 & 1993 \\
\hline 17 & 23 & 681 & 529 & 1985 \\
\hline 17 & 24 & 698 & 576 & 1980 \\
\hline 15 & 25 & 713 & 625 & 1993 \\
\hline 14 & 26 & 727 & 676 & 2001 \\
\hline 14 & 27 & 741 & 729 & 1994 \\
\hline 14 & 28 & 755 & 784 & 1991 \\
\hline
\end{tabular}


Martin B.

\begin{tabular}{|c|c|c|c|c|}
\hline $\mathrm{TC}$ & $r$ & $\Sigma \mathrm{TC}$ & $r^{2}$ & PY \\
\hline 156 & 1 & 156 & 1 & 1983 \\
\hline 74 & 2 & 230 & 4 & 1997 \\
\hline 52 & 3 & 282 & 9 & 1985 \\
\hline 38 & 4 & 320 & 16 & 1983 \\
\hline 35 & 5 & 355 & 25 & 2001 \\
\hline 33 & 6 & 388 & 36 & 1987 \\
\hline 33 & 7 & 421 & 49 & 1985 \\
\hline 30 & 8 & 451 & 64 & 1995 \\
\hline 29 & 9 & 480 & 81 & 1996 \\
\hline 28 & 10 & 508 & 100 & 1984 \\
\hline 24 & 11 & 532 & 121 & 1988 \\
\hline 23 & 12 & 555 & 144 & 1981 \\
\hline 22 & 13 & 577 & 169 & 1999 \\
\hline 20 & 14 & 597 & 196 & 1984 \\
\hline 19 & 15 & 616 & 225 & 1985 \\
\hline 18 & 16 & 634 & 256 & 1986 \\
\hline 16 & 17 & 650 & 289 & 1996 \\
\hline 16 & 18 & 666 & 324 & 1986 \\
\hline 16 & 19 & 682 & 361 & 1985 \\
\hline 16 & 20 & 698 & 400 & 1984 \\
\hline 14 & 21 & 712 & 441 & 1991 \\
\hline 14 & 22 & 726 & 484 & 1984 \\
\hline 11 & 23 & 737 & 529 & 1986 \\
\hline 9 & 24 & 746 & 576 & 1994 \\
\hline 9 & 25 & 755 & 625 & 1989 \\
\hline 9 & 26 & 764 & 676 & 1987 \\
\hline 6 & 27 & 770 & 729 & 1982 \\
\hline 4 & 28 & 774 & 784 & 1992 \\
\hline
\end{tabular}

\section{Narin F.}

$\begin{array}{ccccc}\text { TC } & r & \Sigma \text { TC } & r^{2} & \text { PY } \\ 112 & 1 & 112 & 1 & 1997 \\ 95 & 2 & 207 & 4 & 1987 \\ 86 & 3 & 293 & 9 & 1976 \\ 82 & 4 & 375 & 16 & 1976 \\ 73 & 5 & 448 & 25 & 1977 \\ 71 & 6 & 519 & 36 & 1991 \\ 70 & 7 & 589 & 49 & 1972 \\ 63 & 8 & 652 & 64 & 1985 \\ 59 & 9 & 711 & 81 & 1992\end{array}$




\begin{tabular}{|c|c|c|c|c|}
\hline 55 & 10 & 766 & 100 & 1978 \\
\hline 55 & 11 & 821 & 121 & 1973 \\
\hline 53 & 12 & 874 & 144 & 1975 \\
\hline 52 & 13 & 926 & 169 & 1991 \\
\hline 52 & 14 & 978 & 196 & 1981 \\
\hline 44 & 15 & 1022 & 225 & 1977 \\
\hline 41 & 16 & 1063 & 256 & 1980 \\
\hline 38 & 17 & 1101 & 289 & 2000 \\
\hline 37 & 18 & 1138 & 324 & 1980 \\
\hline 35 & 19 & 1173 & 361 & 1999 \\
\hline 33 & 20 & 1206 & 400 & 1989 \\
\hline 33 & 21 & 1239 & 441 & 1987 \\
\hline 29 & 22 & 1268 & 484 & 1994 \\
\hline 28 & 23 & 1296 & 529 & 1996 \\
\hline 28 & 24 & 1324 & 576 & 1977 \\
\hline 28 & 25 & 1352 & 625 & 1976 \\
\hline 27 & 26 & 1379 & 676 & 1984 \\
\hline 27 & 27 & 1406 & 729 & 1983 \\
\hline 26 & 28 & 1432 & 784 & 1988 \\
\hline 24 & 29 & 1456 & 841 & 1988 \\
\hline 23 & 30 & 1479 & 900 & 1995 \\
\hline 20 & 31 & 1499 & 961 & 1998 \\
\hline 19 & 32 & 1518 & 1024 & 1994 \\
\hline 18 & 33 & 1536 & 1089 & 1980 \\
\hline 18 & 34 & 1554 & 1156 & 1979 \\
\hline 17 & 35 & 1571 & 1225 & 1978 \\
\hline 14 & 36 & 1585 & 1296 & 1996 \\
\hline 13 & 37 & 1598 & 1369 & 1983 \\
\hline 12 & 38 & 1610 & 1444 & 1986 \\
\hline 10 & 39 & 1620 & 1521 & 1977 \\
\hline 10 & 40 & 1630 & 1600 & 1972 \\
\hline 9 & 41 & 1639 & 1681 & 1983 \\
\hline
\end{tabular}

Schubert A.

$\begin{array}{ccccc}\text { TC } & r & \Sigma T C & r^{2} & \text { PY } \\ 124 & 1 & 124 & 1 & 1989 \\ 90 & 2 & 214 & 4 & 2002 \\ 78 & 3 & 292 & 9 & 1986 \\ 59 & 4 & 351 & 16 & 1978 \\ 57 & 5 & 408 & 25 & 1990 \\ 40 & 6 & 448 & 36 & 1979 \\ 33 & 7 & 481 & 49 & 1988 \\ 32 & 8 & 513 & 64 & 1983 \\ 27 & 9 & 540 & 81 & 1988 \\ 27 & 10 & 567 & 100 & 1987 \\ 27 & 11 & 594 & 121 & 1984 \\ 26 & 12 & 620 & 144 & 2000\end{array}$




\begin{tabular}{llllll}
\hline 26 & 13 & 646 & 169 & 1994 \\
23 & 14 & 669 & 196 & 1994 \\
23 & 15 & 692 & 225 & 1987 \\
22 & 16 & 714 & 256 & 1987 \\
19 & 17 & 733 & 289 & 1986 \\
\cline { 1 - 2 } 18 & 18 & 751 & 324 & 2000 \\
\cline { 1 - 2 } 18 & 19 & 769 & 361 & 1993 \\
18 & 20 & 787 & 400 & 1986 \\
18 & 21 & 805 & 441 & 1984 \\
17 & 22 & 822 & 484 & 2001 \\
17 & 23 & 839 & 529 & 1988 \\
17 & 24 & 856 & 576 & 1982 \\
16 & 25 & 872 & 625 & 1982 \\
15 & 26 & 887 & 676 & 2002 \\
14 & 27 & 901 & 729 & 1993 \\
14 & 28 & 915 & 784 & 1989 \\
14 & 29 & 929 & 841 & 1985 \\
13 & 30 & 942 & 900 & 1992 \\
12 & 31 & 954 & 961 & 1996
\end{tabular}

\section{Glänzel W.}

\begin{tabular}{ccccc}
\hline TC & $r$ & $\Sigma T C$ & $r^{2}$ & PY \\
& & & & \\
124 & 1 & 124 & 1 & 1989 \\
54 & 2 & 178 & 4 & 1988 \\
33 & 3 & 211 & 9 & 1988 \\
32 & 4 & 243 & 16 & 1995 \\
32 & 5 & 275 & 25 & 1983 \\
31 & 6 & 306 & 36 & 1995 \\
28 & 7 & 334 & 49 & 1995 \\
27 & 8 & 361 & 64 & 1988 \\
27 & 9 & 388 & 81 & 1987 \\
27 & 10 & 415 & 100 & 1984 \\
26 & 11 & 441 & 121 & 1994 \\
24 & 12 & 465 & 144 & 2001 \\
23 & 13 & 488 & 169 & 1994 \\
23 & 14 & 511 & 196 & 1987 \\
22 & 15 & 533 & 225 & 2002 \\
22 & 16 & 555 & 256 & 1987 \\
20 & 17 & 575 & 289 & 1994 \\
\cline { 1 - 1 } 19 & 18 & 594 & 324 & 1986 \\
\cline { 1 - 2 } 18 & 19 & 612 & 361 & 1994 \\
18 & 20 & 630 & 400 & 1993 \\
18 & 21 & 648 & 441 & 1986 \\
18 & 22 & 666 & 484 & 1984 \\
17 & 23 & 683 & 529 & 2001 \\
17 & 24 & 700 & 576 & 1988
\end{tabular}




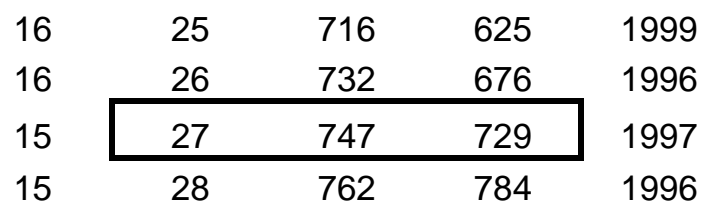

\section{Moed F.H.}

\begin{tabular}{|c|c|c|c|c|}
\hline TC & $r$ & $\Sigma \mathrm{TC}$ & $r^{2}$ & PY \\
\hline 108 & 1 & 108 & 1 & 1985 \\
\hline 56 & 2 & 164 & 4 & 1995 \\
\hline 54 & 3 & 218 & 9 & 1996 \\
\hline 54 & 4 & 272 & 16 & 1995 \\
\hline 49 & 5 & 321 & 25 & 1991 \\
\hline 41 & 6 & 362 & 36 & 1985 \\
\hline 35 & 7 & 397 & 49 & 1991 \\
\hline 31 & 8 & 428 & 64 & 1990 \\
\hline 26 & 9 & 454 & 81 & 2002 \\
\hline 26 & 10 & 480 & 100 & 1989 \\
\hline 24 & 11 & 504 & 121 & 1996 \\
\hline 23 & 12 & 527 & 144 & 1999 \\
\hline 23 & 13 & 550 & 169 & 1998 \\
\hline 22 & 14 & 572 & 196 & 2002 \\
\hline 22 & 15 & 594 & 225 & 1991 \\
\hline 20 & 16 & 614 & 256 & 2001 \\
\hline 20 & 17 & 634 & 289 & 1999 \\
\hline 18 & 18 & 652 & 324 & 1989 \\
\hline 17 & 19 & 669 & 361 & 1985 \\
\hline 15 & 20 & 684 & 400 & 1999 \\
\hline 15 & 21 & 699 & 441 & 1993 \\
\hline 13 & 22 & 712 & 484 & 1998 \\
\hline 13 & 23 & 725 & 529 & 1993 \\
\hline 12 & 24 & 737 & 576 & 2002 \\
\hline 12 & 25 & 749 & 625 & 1993 \\
\hline 9 & 26 & 758 & 676 & 1999 \\
\hline 9 & 27 & 767 & 729 & 1996 \\
\hline 9 & 28 & 776 & 784 & 1996 \\
\hline
\end{tabular}

\section{Leydesdorff L.}

$\begin{array}{ccccc}\text { TC } & r & \Sigma T C & r^{2} & \text { PY } \\ & & & & \\ 79 & 1 & 79 & 1 & 2000 \\ 32 & 2 & 111 & 4 & 1998 \\ 26 & 3 & 137 & 9 & 1986 \\ 24 & 4 & 161 & 16 & 1989 \\ 23 & 5 & 184 & 25 & 1990\end{array}$




\begin{tabular}{ccccc}
\hline 22 & 6 & 206 & 36 & 1987 \\
19 & 7 & 225 & 49 & 1989 \\
17 & 8 & 242 & 64 & 1996 \\
17 & 9 & 259 & 81 & 1991 \\
16 & 10 & 275 & 100 & 1997 \\
15 & 11 & 290 & 121 & 1994 \\
13 & 12 & 303 & 144 & 1994 \\
\cline { 1 - 2 } 13 & 13 & 316 & 169 & 1993 \\
\cline { 1 - 2 } 13 & 14 & 329 & 196 & 1989 \\
11 & 15 & 340 & 225 & 2000 \\
11 & 16 & 351 & 256 & 1993 \\
11 & 17 & 362 & 289 & 1992 \\
10 & 18 & 372 & 324 & 1998 \\
10 & 19 & 382 & 361 & 1997 \\
\cline { 2 - 4 } 9 & 20 & 391 & \multicolumn{3}{|c}{400} & 1992
\end{tabular}

Egghe L.

\begin{tabular}{|c|c|c|c|c|}
\hline TC & $r$ & $\Sigma \mathrm{TC}$ & $r^{2}$ & PY \\
\hline 47 & 1 & 47 & 1 & 1990 \\
\hline 42 & 2 & 89 & 4 & 1985 \\
\hline 37 & 3 & 126 & 9 & 2000 \\
\hline 36 & 4 & 162 & 16 & 1992 \\
\hline 21 & 5 & 183 & 25 & 1992 \\
\hline 18 & 6 & 201 & 36 & 1991 \\
\hline 17 & 7 & 218 & 49 & 1986 \\
\hline 16 & 8 & 234 & 64 & 1995 \\
\hline 16 & 9 & 250 & 81 & 1988 \\
\hline 16 & 10 & 266 & 100 & 1986 \\
\hline 15 & 11 & 281 & 121 & 1993 \\
\hline 13 & 12 & 294 & 144 & 1996 \\
\hline 13 & 13 & 307 & 169 & 1996 \\
\hline 13 & 14 & 320 & 196 & 1990 \\
\hline 13 & 15 & 333 & 225 & 1988 \\
\hline 12 & 16 & 345 & 256 & 2000 \\
\hline 12 & 17 & 357 & 289 & 1994 \\
\hline 12 & 18 & 369 & 324 & 1988 \\
\hline 12 & 19 & 381 & 361 & 1987 \\
\hline 11 & 20 & 392 & 400 & 2000 \\
\hline
\end{tabular}

Rousseau R.

$\begin{array}{rrrrr}\text { TC } & r & \Sigma T C & r^{2} & \text { PY } \\ 25 & 1 & 25 & 1 & 1996\end{array}$




\begin{tabular}{|ccccc}
18 & 2 & 43 & 4 & 2003 \\
18 & 3 & 61 & 9 & 1991 \\
16 & 4 & 77 & 16 & 1995 \\
16 & 5 & 93 & 25 & 1988 \\
15 & 6 & 108 & 36 & 1987 \\
15 & 7 & 123 & 49 & 1992 \\
14 & 8 & 137 & 64 & 1994 \\
13 & 9 & 150 & 81 & 2002 \\
13 & 10 & 163 & 100 & 1999 \\
13 & 11 & 176 & 121 & 1996 \\
13 & 12 & 189 & 144 & 1996 \\
\cline { 1 - 2 } 13 & 13 & 202 & 169 & 1993 \\
\cline { 1 - 2 } 12 & 14 & 214 & 196 & 2000 \\
\cline { 1 - 4 } 12 & 15 & 226 & 225 & 2000 \\
\cline { 1 - 4 } 12 & 16 & 238 & 256 & 1990
\end{tabular}

\section{Ingwersen $\mathbf{P}$.}

\begin{tabular}{|c|c|c|c|c|}
\hline TC & $r$ & $\Sigma \mathrm{TC}$ & $r^{2}$ & PY \\
\hline 120 & 1 & 120 & 1 & 1996 \\
\hline 93 & 2 & 213 & 4 & 1998 \\
\hline 83 & 3 & 296 & 9 & 1997 \\
\hline 79 & 4 & 375 & 16 & 1982 \\
\hline 52 & 5 & 427 & 25 & 2001 \\
\hline 37 & 6 & 464 & 36 & 1997 \\
\hline 31 & 7 & 495 & 49 & 1984 \\
\hline 29 & 8 & 524 & 64 & 1997 \\
\hline 29 & 9 & 553 & 81 & 1987 \\
\hline 19 & 10 & 572 & 100 & 2000 \\
\hline 17 & 11 & 589 & 121 & 1984 \\
\hline 15 & 12 & 604 & 144 & 1996 \\
\hline 14 & 13 & 618 & 169 & 1997 \\
\hline 10 & 14 & 628 & 196 & 2001 \\
\hline 10 & 15 & 638 & 225 & 1992 \\
\hline 8 & 16 & 646 & 256 & 1999 \\
\hline 7 & 17 & 653 & 289 & 1999 \\
\hline 7 & 18 & 660 & 324 & 1995 \\
\hline 6 & 19 & 666 & 361 & 2000 \\
\hline 6 & 20 & 672 & 400 & 1993 \\
\hline 5 & 21 & 677 & 441 & 2000 \\
\hline 3 & 22 & 680 & 484 & 2001 \\
\hline 3 & 23 & 683 & 529 & 2000 \\
\hline 3 & 24 & 686 & 576 & 2000 \\
\hline 3 & 25 & 689 & 625 & 1994 \\
\hline 3 & 26 & 692 & 676 & 1994 \\
\hline 3 & 27 & 695 & 729 & 1992 \\
\hline
\end{tabular}




\section{White H.D.}

\begin{tabular}{ccccc} 
TC & $r$ & $\Sigma T C$ & $r^{2}$ & PY \\
& & & & \\
128 & 1 & 128 & 1 & 1981 \\
106 & 2 & 234 & 4 & 1998 \\
103 & 3 & 337 & 9 & 1989 \\
45 & 4 & 382 & 16 & 1997 \\
37 & 5 & 419 & 25 & 1982 \\
28 & 6 & 447 & 36 & 1981 \\
22 & 7 & 469 & 49 & 1983 \\
21 & 8 & 490 & 64 & 1987 \\
20 & 9 & 510 & 81 & 2001 \\
15 & 10 & 525 & 100 & 1987 \\
14 & 11 & 539 & 121 & 1986 \\
\cline { 1 - 2 } 14 & 12 & 553 & 144 & 1985 \\
\cline { 1 - 2 } 12 & 13 & 565 & 169 & 2003 \\
12 & 14 & 577 & 196 & 1996 \\
12 & 15 & 589 & 225 & 1981 \\
12 & 16 & 601 & 256 & 1981 \\
11 & 17 & 612 & 289 & 2003 \\
10 & 18 & 622 & 324 & 1990 \\
8 & 19 & 630 & 361 & 1986 \\
6 & 20 & 636 & 400 & 2001 \\
5 & 21 & 641 & 441 & 2004 \\
5 & 22 & 646 & 484 & 1986 \\
5 & 23 & 651 & 529 & 1984 \\
5 & 24 & 656 & 576 & 1977 \\
\cline { 1 - 2 } 4 & 25 & 660 & 625 & 2003 \\
4 & 26 & 664 & 676 & 1986 \\
& & & & \\
5 & & & &
\end{tabular}

\section{Conclusions and open problems}

In this paper we studied the g-index being an improvement of the h-index. The g-index $\mathrm{g}$ is the largest rank (where papers are arranged in decreasing order of the number of citations they received) such that the first g papers have (together) at least $g^{2}$ citations. We show that $g \geq h$ and that g always uniquely exists. We present formulae for g in Lotkaian informetrics. We show that 


$$
\begin{aligned}
& g=\left(\frac{\alpha-1}{\alpha-2}\right)^{\frac{\alpha-1}{\alpha}} \mathrm{T}^{\frac{1}{\alpha}} \\
& g=\left(\frac{\alpha-1}{\alpha-2}\right)^{\frac{\alpha-1}{\alpha}} h
\end{aligned}
$$

if these values are $\leq \mathrm{T}$; otherwise $\mathrm{g}=\mathrm{T}$.

Here $\alpha$ is the Lotka exponent and T denotes the total number of sources (in the citation application this means the total number of ever cited papers).

We then calculate the h- and g-indexes of the (still active) Price medallists. Different than in Glänzel and Persson (2005) we do not limit the publication period (except for the fact that we do not use papers older than published in 1972 due to the fact that ISI has no data for them) nor do we limit the topic to informetrics, hence the complete careers (up to 1972) of the Price medallists are considered. It is found that the ranked g-index column resembles more the overall feeling of "visibility" or "life time achievement" than does the ranked h-index column.

We leave open the further exploration of the g-index, including the establishment of the gindex in function of time. In Egghe (2006b) we were able to do this for the h-index based on the cumulative $\mathrm{n}^{\mathrm{nt}}$ citation distribution (see Egghe and Rao (2001)) and in a forthcoming paper we will do the same for the g-index based on a time-dependent Lotkaian theory.

We also leave open the construction of other h- or g-like indexes and the comparison of these new indexes with the h- and g-index. It would also be interesting to work out more practical cases (in other fields) of h- and g-index comparisons. Such case studies can learn a lot on the advantages and/or disadvantages of the h-index and the g-index. 


\section{$\underline{\text { References }}$}

Ball P. (2005). Index aims for fair ranking of scientists. Nature 436, 900.

Braun T., Glänzel W. and Schubert A. (2005). A Hirsch-type index for journals. The Scientist 19(22), 8.

Egghe L. (2005). Power Laws in the Information Production Process: Lotkaian Informetrics. Elsevier, Oxford (UK).

Egghe L. (2006a). An improvement of the h-index: the g-index. Preprint.

Egghe L. (2006b). Dynamic h-index: the Hirsch index in function of time. Preprint.

Egghe L. and Rao I.K.R. (2001). Theory of first-citation distributions and applications. Mathematical and Computer Modelling 34(1-2), 81-90.

Egghe L. and Rousseau R. (1990). Introduction to Informetrics. Quantitative Methods in Library, Documentation and Information Science. Elsevier, Amsterdam (the Netherlands).

Egghe L. and Rousseau R. (2006). An informetric model of the Hirsch index. Preprint.

Glänzel W. (2006a). On the opportunities and limitations of the H-index. Science Focus 1, to appear.

Glänzel W. (2006b). On the H-index - A mathematical approach to a new measure of publication activity and citation impact. Scientometrics 67(2), to appear.

Glänzel W. and Persson O. (2005). H-index for Price medallist. ISSI Newsletter 1(4), 15-18.

Hirsch J.E. (2005). An index to quantify an individual's scientific research output. arxiv:physics/0508025. 